\title{
Microstructural Analyses to Study Ingredient Functionality, Interactions and Quality in Frozen Foods.
}

\section{H. Douglas Goff}

Dept. of Food Science, Univ. of Guelph, Guelph, ON N1G 2W1, Canada, dgoff@uoguelph.ca

During the freezing of foods, ice forms as the temperature is lowered below the equilibrium freezing temperature. With decreasing temperature, the quantity of ice increases and is dictated by the nature of the solutes in solution, as they are freeze-concentrated in a decreasing quantity of water (Fig. 1). The size, morphology and distribution of ice crystals is dependent in large part on the type of freezing process (quiescent versus under shear) and the rate of heat removal [1]. The unfrozen phase can undergo a glass transition due to high concentration and low temperature, and this gives the frozen product much greater storage stability.

During storage at temperatures above the glass transition, especially in fluctuating temperatures, ice crystals undergo recrystallization, in which they grow in size and decrease in number. Several phenomena are involved, including ripening, accretion, melt-diffuse, and shrink-regrow recrystallization. In most cases, these changes can detrimentally affect the quality of the frozen product $[1,2]$.

Polysaccharide stabilizers have been used in frozen food systems for many years, to slow down the process of ice recrystallization and structural changes during frozen storage. Ice cream is one example where their use is of great importance, due to the detrimental effects of recrystallization on texture. Understanding how these polysaccharides function to control ice crystal growth, however, has been challenging, but is of importance to both finding and assessing new ingredients and or modifications to existing food products. Polysaccharides are well known viscosity-enhancers in solution, which occurs through their bulky structure and their ability to interact with each other and with other polymers [3]. As freeze-concentration occurs, viscosity within localized regions (socalled "micro-viscosity") can limit mobility and hence delay ice crystal growth [3]. Through microstructural analyses, two additional phenomena are evident in frozen systems, both of which are also relevant to polysaccharide functionality. The first is cryo-gelation, an inter-molecular interaction induced by freeze-concentration which sets up an element of 3-dimensional macromolecular structure, and the second is incompatibility and phase separation with proteins, if present in the food system, which is also enhanced by freeze-concentration and adds a further element of structure [4].

Various microscopic techniques can be used to explore functionality of polysaccharide stabilizers in frozen systems, including specific staining with cold-stage light or freeze-substitution transmission 
electron microscopy [5] and the use of labeled polysaccharides and cold-stage fluorescence microscopy (Fig. 2) [4]. These techniques augment structural analysis by cryo-scanning electron microscopy [6], to yield valuable information for frozen food formulations and processing.

References

[1] H. D. Goff. Food Research Internat. 25 (1992) 317.

[2] H. D. Goff in Rencontres AGORAL, TEC and DOC, Paris, 1999, 147.

[3] S. Bolliger et al. Internat. Dairy J. 10 (2000) 791.

[4] H. D. Goff et al. Food Hydrocoll., 13 (1999) 353.

[5] A. Regand. M.Sc. Thesis, Univ. of Guelph, 2001.

[6] K. B. Caldwell et al. Food Structure 11 (1992) 1.

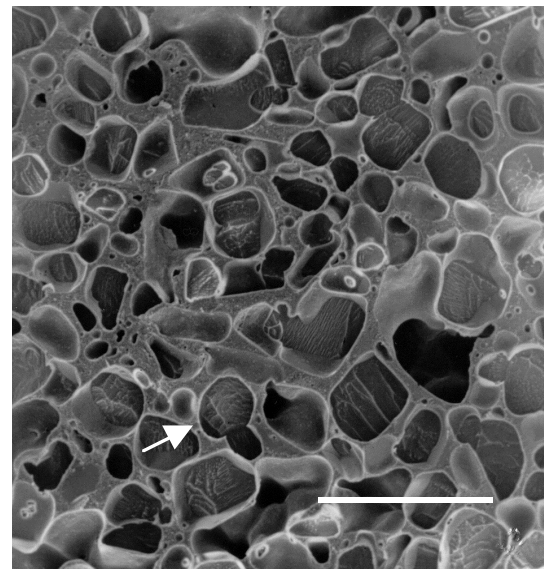

FIG. 1. Cryo-SEM micrograph of ice cream structure, showing ice crystals and the freeze-concentrated unfrozen phase (arrow). Bar $=50 \mu \mathrm{m}$.

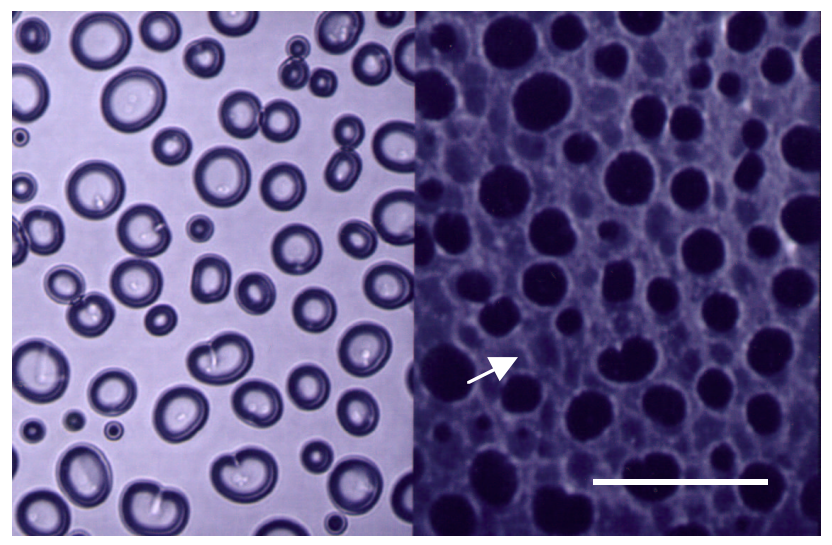

FIG. 2. Brightfield (left) and fluorescence (right) images from the same field of a frozen $40 \%$ sucrose $+0.42 \%$ rhodamine labelled-locust bean gum (LBG) solution after 5 cycles from $-18^{\circ} \mathrm{C}$ to $-10^{\circ} \mathrm{C}$, at $-10^{\circ} \mathrm{C}$. Note the gel-like LBG structure (arrow) in the fluorescence image formed by freezing and temperature cycling. Bar $=50$ $\mu \mathrm{m}$. 